\title{
Base Curve of Progressive Addition Lenses Influences Clear Vision and Stereopsis Area
}

This article was published in the following Dove Press journal: Clinical Optometry

\author{
Go Mawatari' \\ Saeko Uchida ${ }^{2}$ \\ Nobuhisa Nao-i ${ }^{1}$ \\ 'Department of Ophthalmology, Faculty \\ of Medicine, University of Miyazaki, \\ Miyazaki, Japan; ${ }^{2}$ Department of \\ Orthoptics and Visual Sciences, Kyusyu \\ University of Health and Welfare, \\ Nobeoka, Miyazaki, Japan
}

Purpose: Progressive addition lenses (PAL) are effective, particularly for middle-aged and elderly people who require reading spectacles. However, with PALs, peripheral vision may be distorted and blurred because of both the lateral bending of the surface and the effect of unequal bending of the light coming from an off-axis location in the tangential and sagittal directions, which may lead to a decrease in the quality of vision. Till date, no evaluation of PALs has been reported with regard to peripheral and binocular vision. We investigated the influence of highbase-curve PAL on the visual function of binocular vision using a synoptophore.

Methods: The subjects were seven males and 13 females aged 50-79 years with a bestcorrected visual acuity of decimal visual acuity (1.0) or higher in both eyes and addition power of 1.50-2.50 diopters as the inclusion criteria. The study design was a two-group, twoperiod crossover trial. Using a synoptophore, the subjective clear vision area of monocular vision and stereopsis area of binocular vision were measured while wearing conventionalbase curve PAL (4-curve) and high-base-curve PAL (8-curve). HOYALUX RF SPORT 1.6 lenses (HOYA Corporation, Tokyo, Japan) were used for the test PALs.

Results: The clear vision area of monocular vision was significantly wider when wearing the 8 -curve PAL on the temporal side of the right eye $(P=0.02)$, and on the temporal side of the left eye $(P=0.01)$. The stereopsis area of binocular vision was significantly wider in all directions when wearing the 8-curve PAL: right $(P=0.02)$; left $(P=0.03)$; right $15^{\circ}$ upward $(P=0.02)$; and left $15^{\circ}$ upward $(P=0.02)$.

Conclusion: It was clarified that, compared to 4-curve PAL, clear vision and stereopsis areas are wider when wearing 8-curve PAL.

Keywords: aberration, clear vision area of monocular vision, high-base-curve lenses, stereopsis area of binocular vision, progressive addition lenses, synoptophore

\section{Introduction}

Progressive addition lenses (PAL) are effective, particularly for middle-aged and elderly people who require reading spectacles, because with such lenses there is no boundary between distance and near zones. In addition, focus can be continuously adjusted to distant and near targets, which is convenient. However, with PALs, peripheral vision may be distorted and blurred. ${ }^{1,2}$ The distortion comes from variations in the magnification induced by the optical surface. However, the blurred area varied according to the Minkwitz theorem. ${ }^{1}$ There are two ways for theoretically reducing the blurs and distortion: 1) extending the corridor length and 2) reducing the addition power.

Individual aberrations, i.e., astigmatism and field curvature (power error), are considered when lenses are designed. ${ }^{3,4}$ The role of an optical design is to minimize
Correspondence: Nobuhisa Nao-i Department of Ophthalmology, Faculty of Medicine, University of Miyazaki, 5200 Kihara, Kiyotake, Miyazaki 889-1692, Japan Email naoi@med.miyazaki-u.ac.jp 
the aberration-induced reduction of Quality of Vision (QOV). In monofocal lenses, peripheral astigmatism can be reduced by increasing the lens base curve, following Tscherning ellipses, or by introducing opposite surface astigmatism using asphericity in at least one of the surfaces. Such design factors are generally known to improve the comfort of spectacles. ${ }^{5}$ However, with regard to the binocular function, these factors have not been evaluated quantitatively using subjects actually wearing spectacles.

We collected quantitative data from subjects and investigated the influence of high-base-curve PALs on the visual function. The PAL distance zone was investigated considering that PALs are frequently worn when driving a vehicle. ${ }^{6}$

\section{Materials and Methods Subjects}

Twenty subjects (40 eyes; males and females aged 50-79 years) participated in the experiment. The inclusion criteria were a best-corrected visual acuity of decimal visual acuity (1.0) or higher in both eyes, addition power of 1.50-2.50 diopters (D), and wearing PAL for one year or longer. The exclusion criteria were an anisometropia of 2.00 D or more, manifest strabismus, ocular motility disorder, abnormal retinal correspondence, chronic headache, subjective headache, dizziness caused by wearing spectacles, and cognitive impairment or suspected cognitive impairment. All subjects provided informed consent to participate in the study. This study was approved by the Ethics Committee of the Faculty of Medicine, University of Miyazaki (study number: I-0013) and adhered to the Declaration of Helsinki.

\section{Test Spectacles}

The test conventional-base and high-base-curve PALs were prepared after confirming the suitable prescribed power for the subjects. HOYALUX RF SPORT 1.6 lenses (HOYA Corporation, Tokyo, Japan) were used for the test spectacle lenses. The curvature radii of the lens convex surface were $13.3 \mathrm{~cm}$ (4-curve) and $6.6 \mathrm{~cm}$ (8-curve) in the conventional- and high-base-curve PALs, respectively. According to the general custom in the spectacle industry, the lens curve was represented by a crown glass index of 1.523. The corridor length and design of surface astigmatism in the 4- and 8-curve were the same. The ONB-307 (rimless, square type, horizontal boxed lens size: $57 \mathrm{~mm}$; vertical boxed lens size: $30 \mathrm{~mm}$; distance between lenses:
$17 \mathrm{~mm}$; Onbeat Inc., Fukui, Japan) spectacle frame was used for all subjects. The pantoscopic angle was maintained at a uniform $8^{\circ}$ during preparing spectacles, and two experienced opticians adjusted the vertex distance $(12 \mathrm{~mm})$ and eye point.

\section{Study Design and Test Methods}

The study employed was a two-group, two-period crossover trial design. The subjects were allocated to two groups (1:1) by block randomization. For Group 1, measurement was performed in the first and second periods wearing the 8- and 4-curve lenses, respectively. Conversely, with Group 2, measurement was performed in the first and second periods wearing the 4- and 8-curve lenses. A single-blind test was used as the blinding, and the subjects were not informed about the type of spectacle lens curve. Note that there was a 10-minute rest interval between the first and second periods wherein the participants did not wear spectacles. The difference between 4and 8-lens base curves is clear, i.e., the spectacles could be distinguished visually; however, during the study, the spectacles were managed by numbering.

\section{Measurement Items and Methods}

A synoptophore (Clement Clarke Ltd., Harlow, UK; Figure 1) was used to measure the clear vision area of monocular vision (clear vision area) and stereopsis area of binocular vision (stereopsis area) in the first and second periods. In each test, the target movement speed was set to $3 \%$ second. All measurements were performed by a single examiner. To maintain test accuracy, the examiner carefully monitored the rotation of the subject's head and neck during the test.

\section{Measurement of the Clear Vision Area}

Measurement of the clear vision area was performed using self-made Landolt ring target slides. Targets for decimal visual acuity of $0.1-1.0$ were prepared. First, the minimum size of the Landolt ring gap that a subject could distinguish from a primary gaze position was determined. The measurement was performed using the minimum size target slide with one eye covered. Then, the examiner moved the target from the most peripheral lateral region, in which visual recognition was impossible, toward the center. The subject followed the target from the point at which it could be recognized visually. The target is blurry in the aberration region in the periphery of the lens and becomes clear as it moves closer to the frontal clear vision area. The 


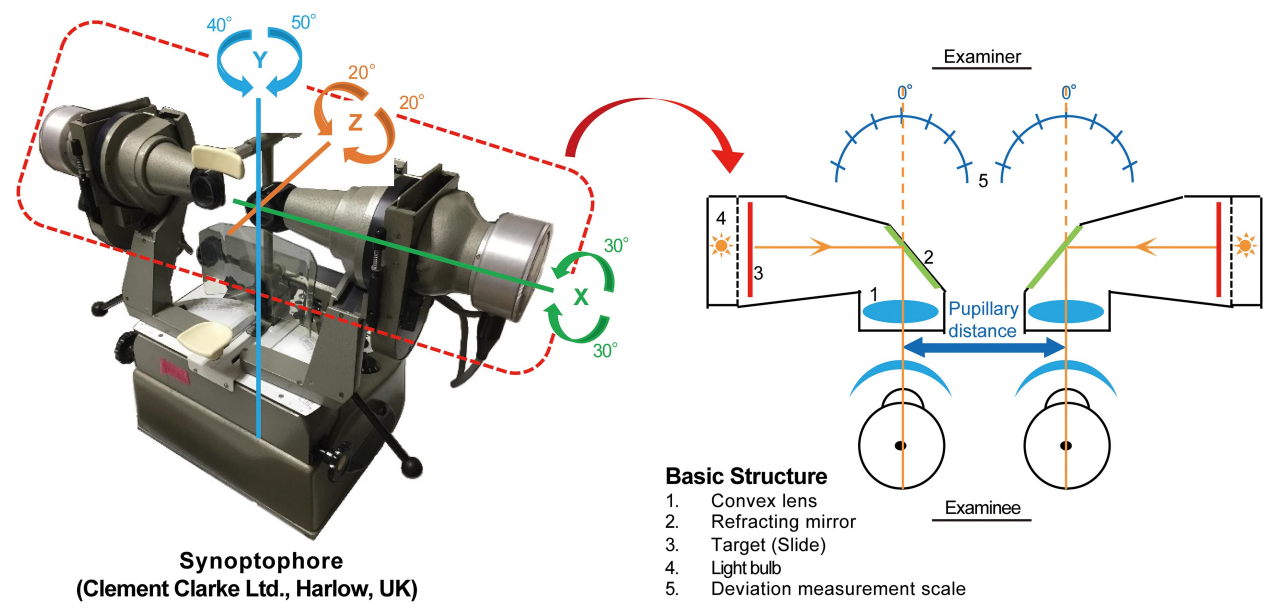

Figure I Basic structure of synoptophore ( $\mathrm{x}$-axis: vertical rotation center; $\mathrm{y}$-axis: horizontal rotation center, $\mathrm{z}$-axis: rotation center). Horizontal and vertical movements and cyclodeviation of the eyeballs, simultaneous perception, fusion amplitude, and stereopsis can be measured quantitatively using a synoptophore. The synoptophore is equipped with a convex lens in the lens barrel, theoretically making it a distance vision-testing device.

angle at which the subject could distinguish the Landolt ring gap was recorded. The position of the gap was changed randomly. The clear vision area was measured three times in four directions (i.e., temporal, nasal, temporal $15^{\circ}$ upward, and nasal $15^{\circ}$ upward) and the mean was adopted.

\section{Measurement of Stereopsis Area}

Measurement of the stereopsis area was performed using Braddick Random Dot graded stereo slides (Clement Clarke Ltd., Harlow, UK). Random dots with a disparity are drawn in each slide for each eye, and stereopsis of the target hidden in the random dots is not possible until binocular fusion is settled. There are four stereo disparities (720, 360, 180, and 90 seconds of arc) and three figures for each stereo disparity in the Braddick Random Dot graded stereo slides. The minimum stereo disparity at which the subject was capable of stereopsis was measured for primary gaze position. Here, slides in which a cross was drawn at the minimum stereo disparity were used. Measurement was performed with binocular vision. After confirming that the subject was capable of stereopsis in front vision, the examiner moved the target from the straight-ahead position toward the periphery in the selected direction. The subject pursued the target moving toward the lateral side, and the angle at which stereopsis broke was recorded. Similar to the clear vision area measurement, the stereopsis area measurement was repeated three times in four directions (i.e., right, left, right $15^{\circ}$ upward, and left $15^{\circ}$ upward), and the mean was adopted as the stereopsis area. The measurement and evaluations were performed similarly in each direction.

\section{Statistical Analysis}

Assuming a minimum difference of $7.5^{\circ}$ (standard deviation: $5^{\circ}$ ) between the two base curves in each direction due to the lack of a previous study, the sample size was calculated to require a minimum of 18 cases with $5 \%$ significance level on both sides and $80 \%$ detection power.

Decimal visual acuity was adopted for visual acuity in all measurements, and the values were converted to logarithm of the minimum angle of resolution (logMAR) and subjected to statistical analysis. In addition, the distance sphere and cylinder powers were converted to their spherical equivalent for statistical analysis.

First, the carryover effect was investigated by applying an analysis of variance (ANOVA) model that included the carryover effect. Then, interaction was investigated using ANOVA that included the interaction between the effect of the lens base curve and the period. Subsequently, the effect of the lens base curve was investigated using an ANOVA model that included the effects of the lens base curve and period. This study was exploratory research, accordingly, we did not perform multiple comparisons. The sex ratio between groups was compared using the $\chi^{2}$ test. The subjects' background (age, visual acuity (distance and near visual acuity), spherical equivalent, addition power, pupillary distance, clear vision area measurement slides, and stereopsis area measurement slides) were compared using an unpaired $t$-test. The result of clear vision and stereopsis area in any corresponding direction were compared using the paired $t$-test. Pearson's correlation coefficient was used to analyze the correlation between the clear or stereopsis vision area 
and the pupillary distance, addition power, and spherical equivalent. The significance level was set to 2 -sided $5 \%$. Note that we used EZR statistical analysis software (ver.1.34; Saitama Medical Center, Jichi Medical University) ${ }^{7}$

\section{Results}

\section{Subjects and Background}

The subjects were seven males and 13 females aged 65.55 \pm 1.61 years on average. No significant difference was noted in the subjects' background between the two groups (Table 1). The range of sphere and cylinder powers was -2.75 to $+2.50 \mathrm{D}$ and 0 to $-2.50 \mathrm{D}$, respectively (Supplementary Table A1).

\section{Clear Vision Area}

The measurement results for the clear vision area in each direction are shown in Table 2. The clear vision area was significantly wider when wearing the 8-curve lens compared with wearing the 4-curve lens in the temporal directions of the right eye, temporal directions of the left eye, and temporal $15^{\circ}$ upward direction of the left eye. In the temporal $15^{\circ}$ upward direction of the right eye, the difference was not significant; however, the area tended to be wider when wearing the 8-curve lens compared with wearing the 4-curve lens. In addition, the area tended to be wider when wearing the 8-curve lens compared with wearing the 4-curve lens. In the nasal direction of the right eye, nasal direction of the left eye, nasal $15^{\circ}$ upward direction of the right eye, and nasal $15^{\circ}$ upward directions of left eyes, however, the differences were not significant.

\section{Stereopsis Area}

The measurement results for the stereopsis area in each direction are shown in Table 2. The stereopsis area was significantly wider when wearing the 8-curve lenses compared with wearing the 4-curve lenses in all directions. A minimum of $13.67^{\circ}$ or wider stereopsis area was acquired when wearing the 8-curve lenses compared with wearing the 4-curve lenses.

\section{Comparison of Clear Vision Area or} Stereopsis Area with Pupillary Distance, Addition Power, and Spherical Equivalent

There was no significant correlation between clear vision area or stereopsis area and pupillary distance, addition power, and spherical equivalent.

\section{Discussion}

In the case of PAL, the clear vision area is limited owing to the surface astigmatism depending on the addition power, corridor length, and effect of the unequal bending of light coming from an off-axis location. In this study, we prepared two designs with same surface astigmatism and different curvatures. Consequently, we confirmed that the high-base-curve PAL is effective in improving visual function through experiments because the clear vision area of the PAL, which is inevitably influenced by surface astigmatism.

A quantitative evaluation of curve lenses when subjects wear the lenses may not only be the factor essential for an evaluation from the optical and engineering perspectives, the visual function perspective may also be important. We measured the highest human binocular function, i.e., stereopsis, from the visual function perspective. Stereopsis is based on the following conditions: 1) the visual fields of the two eyes overlap; 2) manifest strabismus is absent; 3) the decimal visual acuities of the two eyes are more than $(0.8)^{8}$ and aniseikonia (which interferes with binocular single vision) is absent; and 4) the retinal correspondence of the two eyes is normal. ${ }^{9,10}$ To the best of our knowledge, no previous study has used a synoptophore to evaluate the binocular function of spectacle lenses.

\section{Clear Vision Area}

Shimizu reported the range in which the Landolt ring at a decimal visual acuity of 0.8 could be distinguished at a 4-m distance when wearing PAL. ${ }^{11}$ Other than this single report, no study of the clear vision area using PAL has been reported. In that report, the influence of the spectacles fitting condition on the field of fixation was investigated, and the field of right and left fixations under normal fitting was approximately $18^{\circ}$ and $16^{\circ}$, respectively, in binocular vision. The result of wearing the 4-curve lens was approximately $20^{\circ}$ to $15^{\circ}$ at the temporal and nasal directions in monocular vision and showed almost the same results as the previous report. In the current study, we confirmed fitting each time in the tests. In addition, the clear vision area in distance vision could be measured precisely and simply using the synoptophore.

In the spectacle frame used in this study, the lens was wider on the temporal than in the optical center over the nasal side, which may have resulted in a significantly wider optic area in the temporal direction, in which 
Table I Subject Background

\begin{tabular}{|c|c|c|c|c|}
\hline & Total $(n=20)$ & Group I $(n=10)$ & Group2 $(n=10)$ & $\mathbf{P}$ \\
\hline Male/Female (number) & $7 / 13$ & $4 / 6$ & $3 / 7$ & 0.64 \\
\hline Age (years) & $66 \pm 2(50 \sim 77)$ & $68 \pm 2(55 \sim 77)$ & $63 \pm 7$ (50 7I) & 0.12 \\
\hline \multicolumn{5}{|c|}{ Distance visual acuity (logMAR) } \\
\hline RE & $-0.05 \pm 0.04(0 \sim-0.08)$ & $-0.06 \pm 0.03(0 \sim-0.08)$ & $-0.04 \pm 0.04(0 \sim-0.08)$ & 0.18 \\
\hline LE & $-0.06 \pm 0.04(0 \sim-0.08)$ & $-0.06 \pm 0.04(0 \sim-0.08)$ & $-0.06 \pm 0.04(0 \sim-0.08)$ & 0.99 \\
\hline \multicolumn{5}{|c|}{ Near visual acuity (logMAR) } \\
\hline RE & $0.10 \pm 0.11(0.40 \sim 0)$ & $0.14 \pm 0.13(0.40 \sim 0)$ & $0.07 \pm 0.07(0.15 \sim 0)$ & 0.18 \\
\hline LE & $0.09 \pm 0.11(0.30 \sim 0.08)$ & $0.09 \pm 0.13(0.30 \sim 0.08)$ & $0.09 \pm 0.10(0.30 \sim 0)$ & 0.95 \\
\hline \multicolumn{5}{|l|}{ Spherical equivalent (D) } \\
\hline RE & $-0.01 \pm 1.50(-3.00 \sim 2.25)$ & $0.46 \pm 1.25(-1.75 \sim 2.25)$ & $-0.48 \pm 1.64(-3.00 \sim 1.25)$ & 0.17 \\
\hline LE & $-0.05 \pm 1.51(-3.00 \sim 2.25)$ & $0.25 \pm 1.32(-1.75 \sim 2.25)$ & $-0.35 \pm 1.69(-3.00 \sim 2.00)$ & 0.39 \\
\hline \multicolumn{5}{|l|}{ Addition power (D) } \\
\hline RE & $2.14 \pm 0.29(1.50 \sim 2.50)$ & $2.18 \pm 0.24(1.75 \sim 2.50)$ & $2.10 \pm 0.34(1.50 \sim 2.50)$ & 0.57 \\
\hline LE & $2.14 \pm 0.29(1.50 \sim 2.50)$ & $2.18 \pm 0.24(1.75 \sim 2.50)$ & $2.10 \pm 0.34(1.50 \sim 2.50)$ & 0.57 \\
\hline \multicolumn{5}{|l|}{ Pupillary distance (mm) } \\
\hline RE & $31.85 \pm 1.56(29.00 \sim 35.00)$ & $31.7 \pm 1.25(29.00 \sim 33.50)$ & $32.00 \pm 1.87(30.00 \sim 35.00)$ & 0.68 \\
\hline LE & $31.65 \pm 1.63(29.00 \sim 34.50)$ & $31.90 \pm 1.52(29.00 \sim 34.00)$ & $31.40 \pm 1.78(29.50 \sim 34.50)$ & 0.51 \\
\hline \multicolumn{5}{|c|}{ Clear vision area slide (logMAR) } \\
\hline RE & $0.16 \pm 0.07(0.30 \sim 0.05)$ & $0.16 \pm 0.06(0.30 \sim 0.10)$ & $0.15 \pm 0.07(0.22 \sim 0.05)$ & 0.72 \\
\hline LE & $0.19 \pm 0.08(0.30 \sim 0.05)$ & $0.18 \pm 0.08(0.30 \sim 0.05)$ & $0.20 \pm 0.07(0.30 \sim 0.10)$ & 0.52 \\
\hline \multicolumn{5}{|c|}{ Stereopsis area slide (seconds of arc) } \\
\hline & $184.50 \pm 111.09(360 \sim 90)$ & $153.00 \pm 112.65(360 \sim 90)$ & $216.00 \pm 105.64(360 \sim 90)$ & 0.21 \\
\hline
\end{tabular}

Note: Mean \pm SD (range).

Abbreviations: RE, right eye; LE, left eye; logMAR, logarithm of the minimum angle of resolution; $D$, diopter.

peripheral surface of lens has a large aberration. In addition, the difference in the angle formed by the visual axis and lens between the 4- and 8-curve lenses was small on the nasal side, which suggests that the difference in the measurement results was also small. However, the optic area was wider when wearing the 8-curve lens compared with wearing the 4-curve lens in all directions, thereby suggesting that a smaller peripheral astigmatism of the 8-curve lens was also demonstrated in monocular vision.

No significant difference was noted in the refractive power or visual acuity between the two eyes; however, the clear vision area differed between them. Nitta et $\mathrm{al}^{12}$ have reported that the dominant eye interfered with the non-dominant eye on a visual acuity test, and the degree of interference was related to the degree of ocular dominancy. The synoptophore completely separates the vision of the two eyes (i.e., the dichoptic view) using a lens barrel; thus, the influence of the dominant eye on the results is considered. Since the dominant eye was not determined in this study, this remains an issue to investigate in the future.

Shirayanagi reported that super-high-curve single vision spectacle lenses outperformed normal-curve lenses relative to the average power error, astigmatism, distortion, and lateral chromatic aberration. ${ }^{13}$ In the present study, various aberrations, including astigmatism, were reduced more when wearing the 8-curve lens compared to wearing the 4-curve lens, which may have led to the wider optic area when wearing the 8-curve lens compared with wearing the 4-curve lens. Note that the measured value varied markedly 
Table 2 Synoptophore Measurement Items and Results in Each Direction

\begin{tabular}{|c|c|c|c|c|}
\hline & & 4 Curve $\left({ }^{\circ}\right)$ & 8 Curve $\left({ }^{\circ}\right)$ & $P$ \\
\hline \multirow[t]{8}{*}{$\begin{array}{l}\text { Clear vision } \\
\text { area }\end{array}$} & RE) Temporal & $\begin{array}{l}20.28 \pm 7.35(10 \sim 36.33)(95 \% \mathrm{Cl} \\
16.84-23.72)\end{array}$ & $\begin{array}{l}22.30 \pm 6.58(9.67 \sim 37.33)(95 \% \mathrm{Cl} \\
19.2 \mid-25.38)\end{array}$ & 0.02 \\
\hline & RE) Nasal & $\begin{array}{l}\text { I7.97 } \pm 8.44(2.67 \sim 33.67)(95 \% \mathrm{Cl} \\
|4.02-2| .92)\end{array}$ & $\begin{array}{l}18.25 \pm 7.99(2.33 \sim 33.00)(95 \% \mathrm{Cl} \\
|4.5|-2 \mid .99)\end{array}$ & 0.74 \\
\hline & $\begin{array}{l}\text { RE) Temporal } 15^{\circ} \\
\text { upward }\end{array}$ & $\begin{array}{l}19.58 \pm 6.62(10.33 \sim 34.00)(95 \% \mathrm{Cl} \\
16.48-22.68)\end{array}$ & $\begin{array}{l}21.33 \pm 6.45(\mid 0.67 \sim 34.00)(95 \% \mathrm{Cl} \\
|8.3|-24.35)\end{array}$ & 0.14 \\
\hline & RE) Nasal $15^{\circ}$ upward & $\begin{array}{l}|8.1| \pm 8.09(\mid .67 \sim 33.00)(95 \% \mathrm{Cl} \\
|4.33-2| .90)\end{array}$ & $\begin{array}{l}\text { I8.47 } \pm 8.30(3.67 \sim 30.33)(95 \% \mathrm{Cl} \\
\mid 4.58-22.35)\end{array}$ & 0.82 \\
\hline & LE) Temporal & $\begin{array}{l}14.83 \pm 8.74(3.00 \sim 34.33)(95 \% \mathrm{Cl} \\
10.74-18.92)\end{array}$ & $\begin{array}{l}|7.03 \pm 7.8|(I .67 \sim 33.33)(95 \% \mathrm{Cl} \\
\mid 3.38-20.69)\end{array}$ & 0.01 \\
\hline & LE) Nasal & $\begin{array}{l}\text { I6.67 } \pm 7.93(3.33 \sim 35.33)(95 \% \mathrm{Cl} \\
\text { I2.95-20.37) }\end{array}$ & $\begin{array}{l}17.63 \pm 7.33(5.67 \sim 31.67)(95 \% \mathrm{Cl} \\
|4.20-2| .06)\end{array}$ & 0.25 \\
\hline & $\begin{array}{l}\text { LE) Temporal } 15^{\circ} \\
\text { upward }\end{array}$ & $\begin{array}{l}16.55 \pm 6.98(4.33 \sim 30.33)(95 \% \mathrm{Cl} \\
\mid 3.28-19.82)\end{array}$ & $\begin{array}{l}18.83 \pm 6.83(6.33 \sim 32.67)(95 \% \mathrm{Cl} \\
\mid 5.63-22.03)\end{array}$ & 0.0003 \\
\hline & LE) Nasal $15^{\circ}$ upward & $\begin{array}{l}\text { I8.82 } \pm 7.53(2.67 \sim 34.33)(95 \% \mathrm{Cl} \\
\text { I5.29-22.34) }\end{array}$ & $\begin{array}{l}19.37 \pm 6.97(6.67 \sim 31.33)(95 \% \mathrm{Cl} \\
16.10-22.63)\end{array}$ & 0.52 \\
\hline \multirow[t]{4}{*}{$\begin{array}{l}\text { Stereopsis } \\
\text { area }\end{array}$} & Right & $\begin{array}{l}20.48 \pm 4.47(\mid 2.33 \sim 27.00)(95 \% \mathrm{Cl} \\
18.39-22.58)\end{array}$ & $\begin{array}{l}22.98 \pm 3.60(14.00 \sim 28.33)(95 \% \mathrm{Cl}, \\
21.29-24.67)\end{array}$ & 0.02 \\
\hline & Left & $\begin{array}{l}\text { I7.47 } \pm 4.47(9.00 \sim 26.33)(95 \% \mathrm{Cl} \\
\text { I5.37-19.56) }\end{array}$ & $\begin{array}{l}19.78 \pm 4.25(13.67 \sim 28.00)(95 \% \mathrm{Cl} \\
|7.80-2| .77)\end{array}$ & 0.03 \\
\hline & Right $15^{\circ}$ upward & $\begin{array}{l}\mathrm{I} 8.80 \pm 6.57(\mathrm{I} 0.33 \sim 28.33)(95 \% \mathrm{Cl} \\
|5.72-2| .87)\end{array}$ & $\begin{array}{l}21.32 \pm 4.78(14.00 \sim 29.33)(95 \% \mathrm{Cl} \text {, } \\
19.08-23.55)\end{array}$ & 0.02 \\
\hline & Left $15^{\circ}$ upward & $\begin{array}{l}\mid 8.13 \pm 5.39(7.33 \sim 29.00)(95 \% \mathrm{Cl} \\
|5.6|-20.66)\end{array}$ & $\begin{array}{l}20.62 \pm 5.00(\text { ( } 4.67 \sim 29.00)(95 \% \mathrm{Cl}, \\
\text { I8.28-22.95) }\end{array}$ & 0.02 \\
\hline
\end{tabular}

Note: Mean \pm SD (range).

Abbreviations: RE, right eye; LE, left eye.

among subjects in each measurement direction; however, the within-subject variation was small.

\section{Stereopsis Area}

No significant difference was observed between the results of the clear vision and stereopsis areas in any corresponding direction, which suggests that the results of the two optic areas were similar (Table 3). The clear vision area was wider when wearing the 8-curve lenses compared with wearing the 4-curve lenses in all directions, and this suggests that acquiring distinct visual acuity in a wider area when wearing the 8-curve lenses compared with wearing the 4-curve lenses resulted in a wider stereopsis area. These findings suggest that the average power error, astigmatism, distortion, and lateral chromatic aberration are smaller in binocular vision while wearing the 8-curve lenses compared with wearing the 4-curve lenses.
The wraparound frame for high-base-curve lenses has a problem with a face form angle-induced unnecessary prismatic effect in the center and periphery of the lens, which induces visual axis deviation between the two eyes and influences binocular vision negatively. In the two types of lens used in this study, the unnecessary prismatic effect at the optical center of the lens produced by the face form angle was corrected, and the angle formed by the peripheral lens surface and visual line became close to perpendicular due to the curve depth of the 8-curve lens. Accordingly, the unnecessary prismatic effect was reduced. Due to these optical performance characteristics, 8-curve lenses may be advantageous relative to acquiring a wider stereopsis area. The measured values of the stereopsis area showed variation among the subjects in all measurement directions; however, the within-individual variation was small. 
Table 3 Comparison of Stereopsis and Clear Vision Areas

\begin{tabular}{|l|l|l|l|}
\hline \multirow{2}{*}{ Stereopsis Area } & Clear Vision Area & \multicolumn{2}{l|}{} \\
\cline { 3 - 4 } & & 4Curve & 8Curve \\
\hline Right & RE) Temporal & 0.92 & 0.64 \\
& LE) Nasal & 0.05 & 0.004 \\
\hline Left & RE) Nasal & 0.83 & 0.48 \\
\hline Right $15^{\circ}$ upward & LE) Temporal & 0.3 & 0.23 \\
\hline Left $15^{\circ}$ upward & RE) Temporal 15 upward & 0.67 & 0.99 \\
& LE) Nasal 15 upward & 0.99 & 0.28 \\
\hline
\end{tabular}

Abbreviations: RE, right eye; LE, left eye.

In this study, the three following factors could influence the results: pupillary distance, addition power, and spherical equivalent. The addition power is particularly considered to have a strong influence on the results because when the visual line deviates from the corridor, distortion and sway occur, ${ }^{1,3,4}$ i.e., changes in the power from the distant to near zone increases with increased addition power, thereby increasing surface astigmatism in the lens periphery. However, these three factors did not influence the result.

This study has some limitations. First, the used lens model was only one. We compared only 4- and 8-curve lenses, which had a big prospected difference because this study is an exploratory research. The results may be extrapolated to other lens types or base curves because the result is based on Tscherning ellipses; however, this is not clear. Second, when investigating the correlation between the clear vision area or stereopsis area and pupillary distance, addition power, and spherical equivalent, the sample size for disclosing a strong correlation coefficient $r>0.6$ was calculated as 14 cases, and the weak correlation coefficient $r>0.3$ was calculated as 85 cases (5\% significance level and $80 \%$ desired power). The number of samples in this study was 20 cases, which is an insufficient number for examining the weak correlation. Third, only the PAL distance zone was investigated in this study. In the future, an investigation of the near zone may also be necessary because PALs have the strongest aberration area on either side of the near zone. ${ }^{4}$

\section{Conclusions}

The specificity of the 8-curve lens was investigated using the synoptophore. This is the first report to evaluate PALs in terms of binocular function. This study clarified that both clear vision and stereopsis areas are significantly wider when wearing the 8-curve lens compared with wearing the 4-curve lens in one lens model. In addition, the results suggest that 8-curve PAL may be useful relative to acquiring higher visual function. Whether the results can be extrapolated to other base curve values or lens models is not yet clear.

\section{Funding}

This research was supported with a research fund from HOYA Corporation. However, Hoya Corporation had no role in the study design, conduct, analysis, interpretation, and writing of the report.

\section{Disclosure}

The authors report no conflicts of interest in this work.

\section{References}

1. Minkwitz G. On the surface astigmatism of a fixed symmetrical aspheric surface. Opt Acta. 1963;10:223-227. doi:10.1080/713817794

2. Sheedy JE, Campbell C, King-Smith E, Hayes JR. Progressive powered lenses: the Minkwitz theorem. Optom Vis Sci. 2005;82:916-924. doi:10.1097/01.opx.0000181266.60785.c9

3. Hua Q. Optical design of ophthalmic lens and QOV. $J$ Eye. 2007;24:1163-1172.

4. Charman WN. Development in the correction of presbyopia I: spectacle and contact lenses. Ophthalmic Physiol Opt. 2014;34:8-29. doi:10.1111/opo.12091

5. Tscherning's Ellipses. Mo J. The Principles of Ophthalmic Lenses. 5th ed. Godmersham, Canterbury: ABDO college of Education; 2016:354.

6. Kawahara T. Spectacles and visual line. J Eye. 2013;30:1053-1060.

7. Kanda Y. Investigation of the freely available easy-to-use software 'EZR' for medical statistics. Bone Marrow Transplant. 2013;48:452-458. doi:10.1038/bmt.2012.244

8. Hirai Y, Awaya S. Studies on visual acuity and stereo acuity. Folia Ophthalmic Japonica. 1958;36:1524-1531.

9. Asakawa K, Uozato $\mathrm{H}$. The necessary conditions and permissible values for maintaining stereoacuity. J Ophthalmol Opt Soc Jpn. 2011;32:3-7. 
10. Yagasaki T. Clinical artifacts in stereopsis testings. Neuro Ophthalmol Jpn. 2006;23:416-427.

11. Shimizu T. Points to keep in mind for lens fitting -change in field of fixation due to fitting condition of progressive addition lenses. Jpn J Vis Sci. 2002;23:54-62.

12. Nitta M, Shimizu K, Niida T. The influence of ocular dominance on monovision -the influence of strength of ocular dominance on visual functions. J Jpn Ophthalmol Soc. 2007;111:441-446.
13. Shirayanagi M. Optical performance of super-high-curve single-vision spectacle lenses. Jpn $J$ Optom Ophthalmic Sci. 2017;21(1):37-45.
Clinical Optometry

\section{Publish your work in this journal}

Clinical Optometry is an international, peer-reviewed, open access journal publishing original research, basic science, clinical and epidemiological studies, reviews and evaluations on clinical optometry. All aspects of patient care are addressed within the journal as well as the practice of optometry including economic and business analyses. Basic and clinical research papers are published that cover

Submit your manuscript here: https://www.dovepress.com/clinical-optometry-journal
Dovepress

all aspects of optics, refraction and its application to the theory and practice of optometry. The manuscript management system is completely online and includes a very quick and fair peer-review system, which is all easy to use. Visit http://www.dovepress.com/ testimonials.php to read real quotes from published authors. 